\title{
Aortic dissection type A after supra-aortic debranching and implantation of an endovascular stent-graft for type B dissection: A word of caution
}

Payam Akhyari, MD, ${ }^{\text {a }}$ Hiroyuki Kamiya, MD, ${ }^{\mathrm{a}}$ Tobias Heye, MD, ${ }^{\mathrm{b}}$ Artur Lichtenberg, MD, ${ }^{\mathrm{a}}$ and Matthias Karck, MD,

Heidelberg, Germany

Combined procedures involving an endovascular stenting technique and transposition of the aortic arch vessels have been proposed for the treatment of aneurysms or chronic dissections involving the descending thoracic aorta and the

From the Departments of Cardiac Surgery ${ }^{\mathrm{a}}$ and Radiology, ${ }^{\mathrm{b}}$ Heidelberg University Hospital, Heidelberg, Germany.

Received for publication July 22, 2008; accepted for publication Oct 4, 2008. Address for reprints: Payam Akhyari, MD, Department of Cardiac Surgery, University

Clinic Heidelberg, Im Neuenheimer Feld 110, 69120 Heidelberg (E-mail: payam. akhyari@med.uni-heidelberg.de).

J Thorac Cardiovasc Surg 2009;137:1290-2

$0022-5223 / \$ 36.00$

Copyright (C) 2009 by The American Association for Thoracic Surgery doi:10.1016/j.jtcvs.2008.10.006 aortic arch. This novel concept abandons the use of extracorporeal circulation and can be applied without a full-length sternotomy. ${ }^{1}$ Reductions in surgical trauma and periprocedural morbidity and mortality have therefore been proposed as advantages for this novel technique. ${ }^{2}$ Here we report on 2 cases of Stanford type A aortic dissection that occurred during supra-aortic debranching and endovascular stenting of the aortic arch and the descending aorta.

\section{CLINICAL SUMMARY}

In April 2008, 2 patients, both with no signs of Marfan syndrome, were admitted to our department with type A aortic dissection.
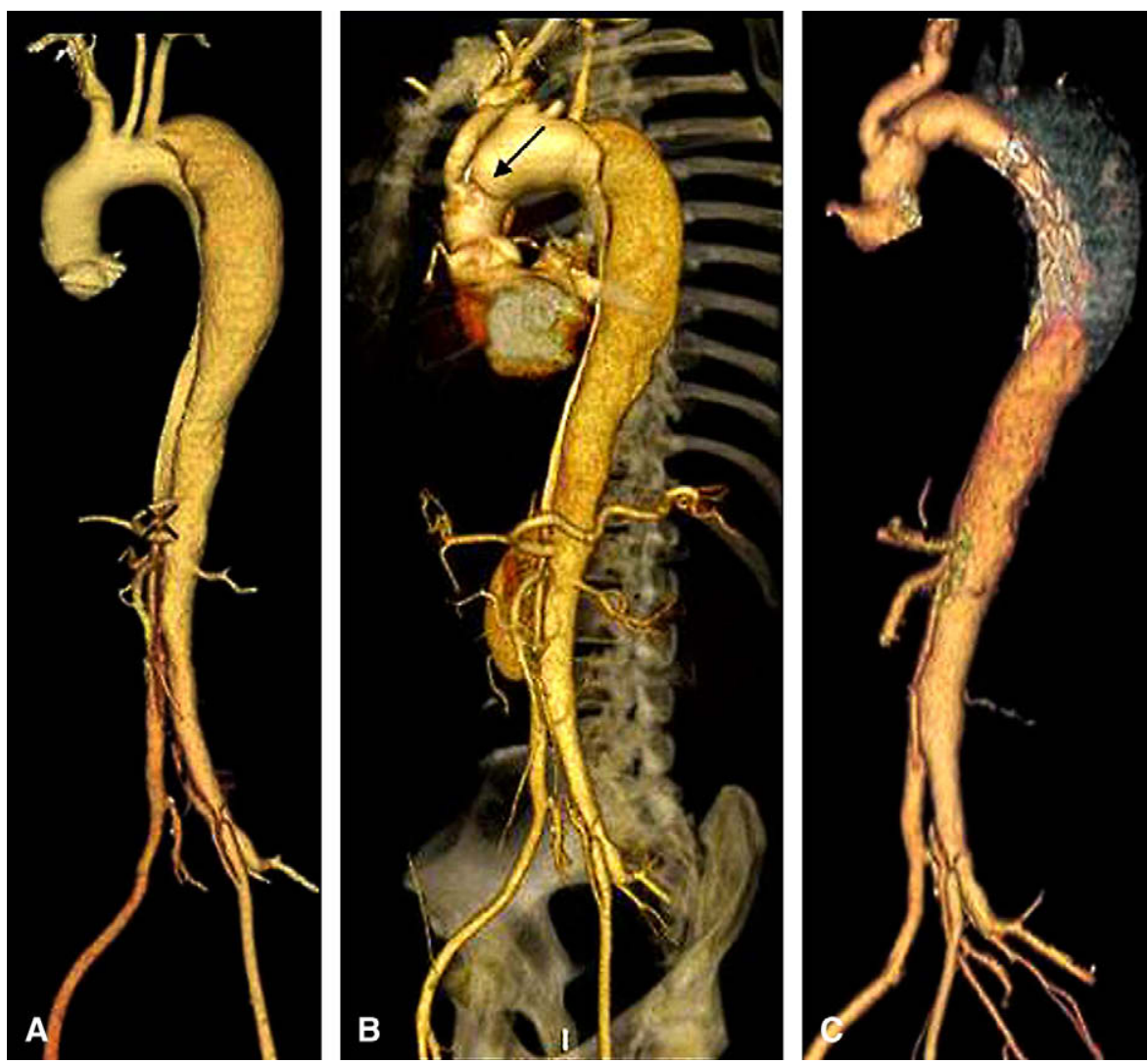

FIGURE 1. A, Three-dimensional reconstruction of computed tomographic angiogram demonstrating initial diagnosis of aortic dissection type B in patient 1. B, After debranching of aortic arch vessels, aortic dissection type A developed (arrow indicates proximal extension of dissection membrane). C, Subsequent correction was performed by heart surgery involving supracoronary replacement of the ascending aorta and the aortic arch, with reinsertion of the debranching prosthesis and addition of a hybrid prosthesis to replace the descending aorta (frozen elephant trunk technique). 


\section{Patient 1}

A 49-year-old man with an expanding chronic type B aortic dissection (maximum diameter $6.0 \mathrm{~cm}$; Figure 1, $A$ ) underwent a prosthetic bypass from the ascending aorta to the innominate artery with a crossover bypass to the left subclavian artery and insertion of the left common carotid artery into the crossover bypass through a partial sternotomy. The postoperative course was complicated by a lymphatic fistula, necessitating operative revision, and by a mild cerebral ischemia with weakness of the right arm. Computed tomographic (CT) scan on the 6th postoperative day showed a retrograde dissection of the ascending aorta. Because of these adverse events, endovascular stenting was postponed, and the patient was discharged home with an aggressive antihypertensive regimen, returning at 3 months with persistent type A aortic dissection on CT scan and intermittent thoracic pain (Figure 1, B). He was referred to our department and underwent an open procedure (through a median sternotomy and with a heart-lung machine) in which the ascending aorta and the aortic arch were replaced, with reinsertion of the debranching prosthesis. The descending aorta was replaced with hybrid prosthesis (frozen elephant trunk technique). The patient was discharged from the intensive care unit on the postoperative day 1 with an uneventful consecutive course. At 1-month follow-up, he was seen in our outpatient clinic with no major complaints, and CT scan revealed unremarkable postoperative findings (Figure 1,C).

\section{Patient 2}

A 70-year-old man with an expanding type B dissection (maximum diameter $6.5 \mathrm{~cm}$ ) underwent a retrosternal crossover bypass from the right to the left subclavian artery with the insertion of the left common carotid artery into the bypass vessel. One week later, an endovascular stenting of the descending aorta extending from the left common carotid artery to the level of the diaphragm was performed. In-hospital course was complicated by pneumonia, acute renal failure on a background of chronic renal failure, and therapeutically relevant depression. Initial CT scan revealed no thoracic dissection membrane and thrombosis of the persistent portion of the descending aorta. At 6-month follow-up, a type A aortic dissection with retrograde expansion to the valvular level was observed, which led to the patient's admission to our department (Figure 2, A). During subsequent surgery, the supracoronary ascending aorta was replaced and the aortic valve was reconstructed. The distal anastomosis between the vascular graft and the stent-graft was performed with hypothermic circulatory arrest and selective antegrade cerebral perfusion. The postoperative course was uneventful (Figure 2,B).

\section{DISCUSSION}

The elephant trunk technique, introduced by Borst and coworkers $^{3}$ in 1984 , has been favored by a large number

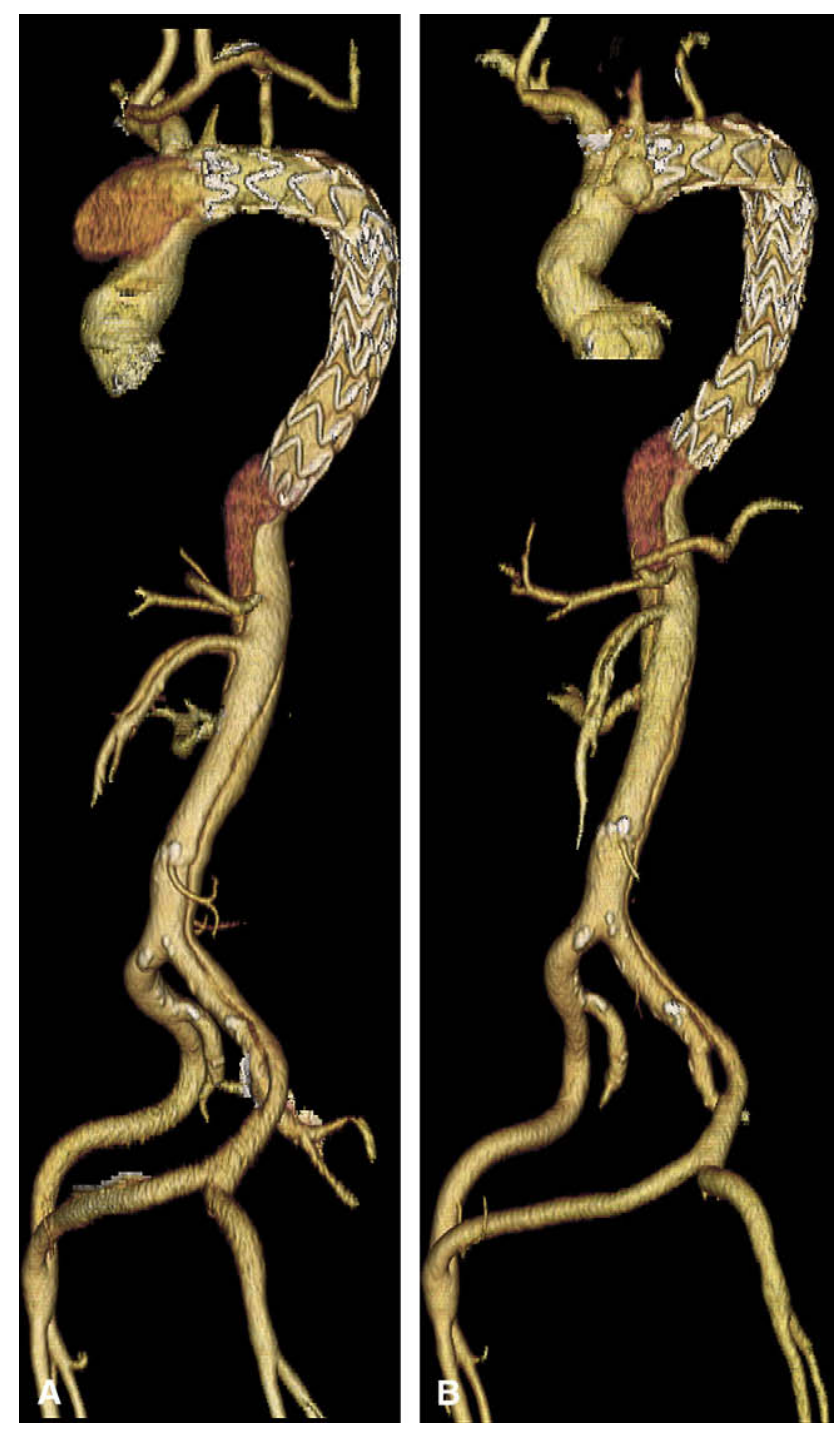

FIGURE 2. A, Three-dimensional reconstruction of computed tomographic angiogram from patient 2 after initial debranching of aortic arch vessels, endovascular stenting of descending aorta and aortic arch, and development of type A aortic dissection with retrograde expansion to level of aortic valve. B, Postoperative result after reconstruction of aortic valve and replacement of ascending aorta with distal graft anastomosis to stentgraft.

of institutions in the treatment of aneurysms involving the aortic arch and the descending aorta. Modifications to this technique have added to feasibility and safety of this procedure. $^{4}$ Recently, endovascular stenting of the aortic arch after transposition of the aortic arch branches has been introduced for treatment of high-risk patients with complex aortic pathology with expected poor outcome after conventional surgery. ${ }^{1}$ Currently reported mortalities with this new approach, however, remain comparable to or even exceed those reported for the conventional approach with the elephant trunk technique and its modern variants. ${ }^{5}$ 
Here we describe 2 patients in whom type A aortic dissection developed during the staged approach of supra-aortic debranching and implantation of an endovascular stentgraft. The most likely mechanism involved was either injury induced by partial clamping of the ascending aorta or an aortic tear by undue radial forces evoked through deployment of the stent-graft in the aortic arch.

Both cases emphasize that despite preliminary encouraging results and in view of different therapeutic options that are available today, including the frozen elephant trunk technique, cautious patient selection remains mandatory for supra-aortic debranching and implantation of a stent-graft into the aortic arch. Procedure-related risks deserve further attention and need to be reevaluated.

\section{References}

1. Schumacher H, Bockler D, Bardenheuer H, Hansmann J, Allenberg JR. Endovascular aortic arch reconstruction with supra-aortic transposition for symptomatic contained rupture and dissection: early experience in 8 high-risk patients. $J$ Endovasc Ther. 2003;10:1066-74.

2. Shah A, Coulon P, de Chaumaray T, Rosario R, Khanoyan P, Boukhris M, et al. Novel technique: staged hybrid surgical and endovascular treatment of acute Type A aortic dissections with aortic arch involvement. J Cardiovasc Surg (Torino). 2006;47:497-502.

3. Borst HG, Walterbusch G, Schaps D. Extensive aortic replacement using "elephant trunk" prosthesis. Thorac Cardiovasc Surg. 1983;31:37-40.

4. Karck M, Chavan A, Hagl C, Friedrich H, Galanski M, Haverich A. The frozen elephant trunk technique: a new treatment for thoracic aortic aneurysms. J Thorac Cardiovasc Surg. 2003;125:1550-3.

5. Karck M, Kamiya H. Progress of the treatment for extended aortic aneurysms; is the frozen elephant trunk technique the next standard in the treatment of complex aortic disease including the arch? Eur J Cardiothorac Surg. 2008; 33:1007-13 